\title{
Segmentação do corpo lúteo em imagens de ultrassom Doppler por meio de CNN com a finalidade de viabilizar o diagnóstico precoce de prenhez em vacas
}

\author{
Thiago M. dos Santos Dias ${ }^{1}$, Cristiane de F. dos Santos ${ }^{2}$, \\ Hugo Jaime. M. C. Peron ${ }^{3}$, Fabrício C. dos Santos $^{4}$ \\ ${ }^{1}$ Instituto Federal Goiano - Campus Urutaí \\ ${ }^{2}$ Núcleo de Informática - Instituto Federal Goiano - Campus Urutaí. \\ ${ }^{3}$ NAPER - Instituto Federal Goiano - Campus Urutaí. \\ ${ }^{4}$ NAPER - Instituto Federal Goiano - Campus Urutaí. \\ ${ }^{1}$ thiagomdias2203@gmail.com \\ ${ }^{2}$ cristiane.santos, ${ }^{3}$ hugo.peron, ${ }^{4}$ fabricio.carriaodifgoiano.edu.br
}

\begin{abstract}
The early determination of pregnancy in cows using ultrasound can generate great savings and increase herd production. Observing the evidence that perfusion in the corpus luteum provides a good parameter to determine early pregnancy, this study aims to perform the segmentation of images from Doppler applied to the reproductive system of bovines, in order to enable early diagnosis of pregnancy, since the calculation of the perfusion area in Doppler ultrasound is trivial in face of a segmented corpus luteum. To perform the segmentation task, a CNN with UNet architecture was developed, resulting in an accuracy of 0.8847 in the set of test images.
\end{abstract}

Resumo. A determinação precoce da prenhez em vacas utilizando ultrassonografia pode gerar grande economia e aumento na produção de rebanhos. Observando as evidências de que a perfusão no corpo lúteo fornece um bom parâmetro para determinar à gestação de maneira precoce, este trabalho tem o objetivo de realizar a segmentação de imagens provenientes de doppler aplicados ao aparelho reprodutor de bovinos, de maneira a viabilizar o diagnóstico precoce da prenhez, uma vez que o cálculo da área de perfusão em ultrassom doppler é trivial diante de um corpo lúteo segmentado. Para a realização da tarefa de segmentação foi desenvolvida uma CNN com arquitetura UNet resultando em uma accuracy de 0.8847 no conjunto de imagens teste.

\section{Introdução}

O processamento digital de imagem é uma área do conhecimento que atende diversas áreas, principalmente no sentido de automatizar processos, como por exemplo, a análise de recursos naturais e meteorologia por meio de imagens de satélites, análise de imagens biomédicas e obtenção de imagens médicas por ultrassom, radiação nuclear ou técnicas de tomografia computadorizada. A medicina e a medicina veterinária podem se beneficiar enormemente do uso de processamento de imagens em função do amplo uso de imagens com a finalidade de diagnóstico, por meio de métricas de calibragem, correção e remoção 
de ruídos, além de um realce que visa melhorar a qualidade de imagem. Com o uso de processamento de imagens, os profissionais da área podem contar com informações mais completas obtidas por meio de técnicas voltadas para à análise de imagens, que são capazes de extrair informações de área, forma, e ainda separar uma região de interesse, dentre outros [Gonzalez and Woods 2010]. Neste contexto, destaca-se o uso de imagens provenientes de ultrassonografia.

De acordo com [Rollim 2017] o modo Doppler permite a avaliação do fluxo nos vasos sanguíneos em situações diversas, como por exemplo na investigação de estruturas reprodutivas, à fim de se obter informações tais como à vascularização uterina em suas diversas fases e apresentações (fase do ciclo estral, gravídico, pré-ovulatório, vascularização do corpo lúteo, perfusão do corpo lúteo, lise do corpo lúteo etc). Segundo [Pugliesi et al. 2017] "os equipamentos Doppler usam como base a diferença entre a frequência das ondas refletidas em estruturas que estão se movimentando com a frequência emitida pelo transdutor, resultando assim no deslocamento de frequência Doppler ou 'Doppler shift'.'A movimentação das células vermelhas no sangue é a responsável por promover uma diferença positiva ou negativa, ou seja, sentido favorável ou contrário ao transdutor, assim, é possível obter informação sobre o fluxo sanguíneo além das habituais imagens dos tecidos e orgãos.

O uso da ultrassonografia para diagnosticar a gestação precocemente, ou seja, na segunda e terceira semanas de gestação foi inicialmente avaliado por [Kastelic et al. 1989]. [Embrapa 2019] e [Pugliesi et al. 2017] falam do uso do Doppler para a detecção de gestação precocemente com base na avaliação da perfusão no corpo lúteo, conforme [Pugliesi et al. 2017] o diagnóstico de gestação pode ser realizado a partir de 28 dias. O ultrassom é utilizado no diagnóstico e acompanhamento de gestação, monitoramento da dinâmica folicular e avaliação da ciclicidade da fêmea. A Figura 1 apresenta uma imagem obtida por meio de doppler pelo Núcleo Avançado de Pesquisa e Extensão de Ruminantes (NAPER).

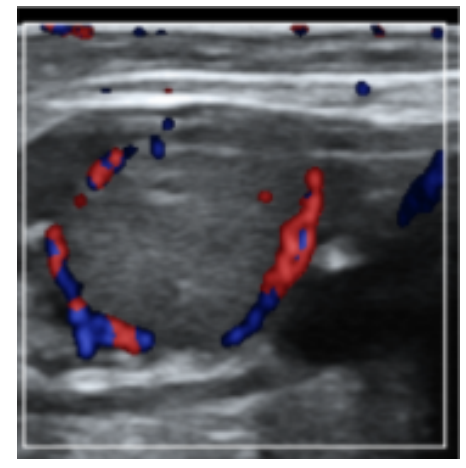

Figura 1. Perfusão em ovário.

Considerando que em programas de Inseminação Artificial em Tempo Fixo (IATF) e da qualidade de receptoras para receberem embriões em programas de Transferência de Embriões em Tempo Fixo (TETF) é importante a obtenção de prazos mais curtos no intervalo de inseminações, a confirmação da não gestação permite a ressincronização precoce, gerando maior produtividade. Segundo a [Embrapa 2019], é possível reduzir o intervalo entre inseminações em até 20 dias com a detecção precoce de prenhez por meio do ultrassom Doppler. Na prática, essa redução pode ser medida em termos de litros 
de leite produzidos à mais: um animal que produz 30 litros de leite por dia produzirá 600 litros à mais até o fim da lactação, e em um rebanho formado por 100 vacas isso significa 60 mil litros de leite [Embrapa 2019]. Deve-se mencionar que a ultrassonografia apresenta significativas vantagens: é uma técnica não invasiva, simples de ser efetuada, segura para o operador e para o animal [Rollim 2017]. Outro ponto importante é que reduzir o intervalo de inseminações pode gerar ganhos tanto no gado de leite quanto em gado de corte.

Ultrassom doppler é aplicado em contextos semelhantes, por exemplo, [Pozor and McDonnell 2004] utiliza a ultrassonografia Doppler colorida para fazer a avaliação da vasculatura do testículo do cavalo e assim obter valores de referência para medidas de fluxo sanguíneo na artéria testicular destes animais. Já [Cheung et al. 2020] realiza a avaliação da perfusão placentária em mulheres no segundo trimestre de gestação, utilizando a ultrassonografia $3 D$ power Doppler. As imagens são segmentadas de forma manual e unidas por meio de técnicas de registro de imagem usando coordenadas de pares selecionadas manualmente. Um outro estudo que apresenta semelhança com o presente trabalho é apresentado por [Rodríguez Alvarez et al. 2018], em que é possível estimar a condição corporal em vacas a partir de imagens de profundidade usando Convolutional Neural Network (CNN). Segundo [Rodríguez Alvarez et al. 2018] é utilizado um método chamado Body Condition Score (BCS) para estimar as reservas de gordura corporal e o balanço energético acumulado das vacas, então é proposto um sistema baseado em CNNs para melhorar a estimativa geral automática de BCS, onde o sistema vai depender de imagens de entrada, em seguida elas são utilizadas para treinar e validar um modelo de CNN.

É utilizado também a Ultrassonografia Doppler para avaliar a perfusão sanguínea da parede folicular e do parênquina luteal, com o objetivo de avaliar o efeito superovolutatório do extrato de pintuitária equina (EPE) no fluxo sanguíneo folicular e luteal em éguas, proporcionando um recrutamento maior de folículos presentes no dia da indução da ovulação [Medeiros et al. 2017]. Outro trabalho que faz o estudo do corpo lúteo [Trevisol 2011], em que objetivo é avaliar a vascularização e volume do corpo lúteo de vacas Caracu adultas após a indução da ovulação com dois análogos distintos de GnRH. Sugerindo que altas concentrações de GnRH elevam as concentrações de hormônio luteinizante resultando na maior vascularização do futuro corpo lúteo.

Há evidências bastante sólidas que sugerem à eficiência da utilização do doppler na determinação precoce da prenhez em bovinos, segundo [Pugliesi et al. 2017], há "uma alta acurácia e sensibilidade quando a ultrassonografia Doppler é empregada para avaliar a funcionalidade do corpo lúteo e diagnosticar a gestação aos 20-22 dias". Diversos estudos podem ser encontrados aplicados a outros tipos de rebanhos enquanto que em bovinos, observa-se a aplicação relativamente recente [Pugliesi et al. 2017]. No entanto, a análise da área de perfusão é ainda subjetiva, e pode resultar em diferentes diagnósticos conforme o profissional que está avaliando e varia também em função da correta determinação das estruturas. Por isso, neste artigo propõe-se o uso de técnicas de processamento de imagens para segmentar imagens de ultrassom de maneira automática, permitindo a quantificação dos níveis de perfusão.

Diante do exposto, o presente artigo se justifica pois tem o potencial de auxiliar na automação do processo de diagnosticar a prenhez em vacas precocemente, uma vez que 
à delimitação da região de interesse por meio de segmentação de imagens torna possível o uso de técnicas de processamento de imagens para quantificar a área de perfusão dando mais segurança ao médico veterinário no diagnóstico da prenhez.

\section{Background}

Alguns conceitos devem ser discutidos antes que se possa de fato abordar a solução proposta. Nas seções a seguir estes conceitos são apresentados.

\subsection{CLAHE}

Um histograma corresponde a uma distribuição discreta de probabilidades de intensidade, assim, a equalização do histograma é uma técnica para melhorar o contraste da imagem pois expande a distribuição dos níveis de intensidade. Contudo, a expansão simultânea de todos os níveis de intensidade pode adicionar intensidades nas extremidades superior e inferior do histograma, ocasionando superexposição ou destaque para aspectos indesejados [Gonzalez and Woods 2000]. A equalização adaptativa do histograma evita isso pois divide a imagem em subimagens, gerando-se histogramas separados para cada uma, e suas respectivas equalizações. No entanto, esse método também apresenta falhas, pode ocorrer o aumento do ruído, sendo que o mesmo é um problema frequente em imagens médicas. CLAHE resolve isso ao utilizar um limite de corte para limitar a altura do histograma antes de calcular a função de distribuição cumulativa. O último passo do CLAHE é fazer com que cada histograma de subimagem seja reorganizado de acordo com uma distribuição: uniforme, Rayleigh ou exponencial [Ma et al. 2017]. O método CLAHE compreende as seguintes etapas:

1. Divida a imagem original em $M \times N$ blocos.

2. Calcule um histograma para cada região com base nos níveis de cinza da imagem.

3. Calcule um histograma de contraste limitado para cada região:

3.1. Calcule o número médio de pixels utilizando o número de níveis de cinza $N_{\text {gray }}$ da região e o número de pixels dentro do bloco de dimensões $X \times Y$. $N_{\text {avg }}=\left(N_{X} \times N_{Y}\right) / N_{\text {gray }}$

3.2. Considerando que $N_{C L}$ é o atual clip limit, e $N_{\text {clip }}$ é o clip limit normalizado em um intervalo de $[0,1]$. Corte os pixels se o número de pixels é maior que $N_{C L}$ :

3.2.1. Calcule o número total de pixels a serem cortados $N_{\sum \text { clip }}$ e a média de pixels restantes $N_{\text {avggray }}=N_{\sum \text { clip }} / N_{\text {gray }}$

3.2.2. Dado que $H_{\text {region }}(i)$ é o histograma original e $H_{\text {region_clip }(i)}$ é o histograma recortado de cada região no i-ésimo nível de cinza, a regra de corte do histograma é dada por a seguir:

$$
\begin{aligned}
& \text { Se } H_{\text {region }(i)}>N_{C L} \text { OU } H_{\text {region }}(i)+N_{\text {avggray }}>N_{C L} \text { então } \\
& H_{\text {region_clip }(i)=N_{C L}}
\end{aligned}
$$

Senão

$H_{\text {region_clip }(i)=H_{\text {region }}(i)+N_{C L}}$

4. Dado que $N_{\text {remain }}$ é o número restante de cortados pixels, Step é um número inteiro positivo maior que ou igual a 1, redistribua os pixels restantes usando: Step $=N_{\text {gray }} / N_{\text {remain }}$

4.1. Progrida do nível de cinza mínimo ao máximo por meio do Step. Se o número de pixels no nível de cinza for menor que $N_{C L}$, distribua um pixel para o nível de cinza. 
4.2. Se todos os pixels não foram distribuídos ao final da pesquisa, calcule um novo Step e inicie uma nova rodada de pesquisa. Pare quando todos os pixels restantes forem distribuídos.

5. Aumente os valores de intensidade em cada região usando uma transformação, por exemplo, Rayleigh.

6. Reduza os efeitos que mudam abruptamente usando o alongamento de contraste linear.

$$
y(i)=\frac{x(i)-x_{\min }}{x_{\max }-x_{\min }}
$$

7. Elimine artefatos de limite calculando a atribuição de nível de cinza de pixels por meio de interpolação bi-linear.

\subsection{Redes neurais}

Redes neurais artificiais surgiram com o objetivo de simular o processamento da informação no cérebro humano e vêm sendo melhoradas ao longo dos anos. Um dos avanços na área é relativo às redes neurais convolucionais $(\mathrm{CNN})$. $\mathrm{O}$ primeiro desafio em CNNs é conseguir uma quantidade relativamente grande de exemplos rotulados para a etapa de treinamento: é preciso fornecer imagens e informações dizendo do que se trata a imagem para que a rede possa aprender e generalizar, classificando novas imagens que sejam submetidas a rede.[Santos et al. 2005].

Redes Neurais Convolucionais são um tipo de RNA especializado para o processamento de dados de entrada que possuem um formato de matriz, usualmente utilizadas para o processamento de informações visuais. O que caracteriza esse tipo de rede é ser composta basicamente de camadas convolutivas, que processam as entradas considerando campos receptivos locais. Em outras palavras, as CNNs são uma espécie de extrator de características automático que antecede uma RNA do tipo Multilayer Feedforward para classificação do resultado em classes distintas. A Arquitetura de uma CNN é mostrada na Figura 2.

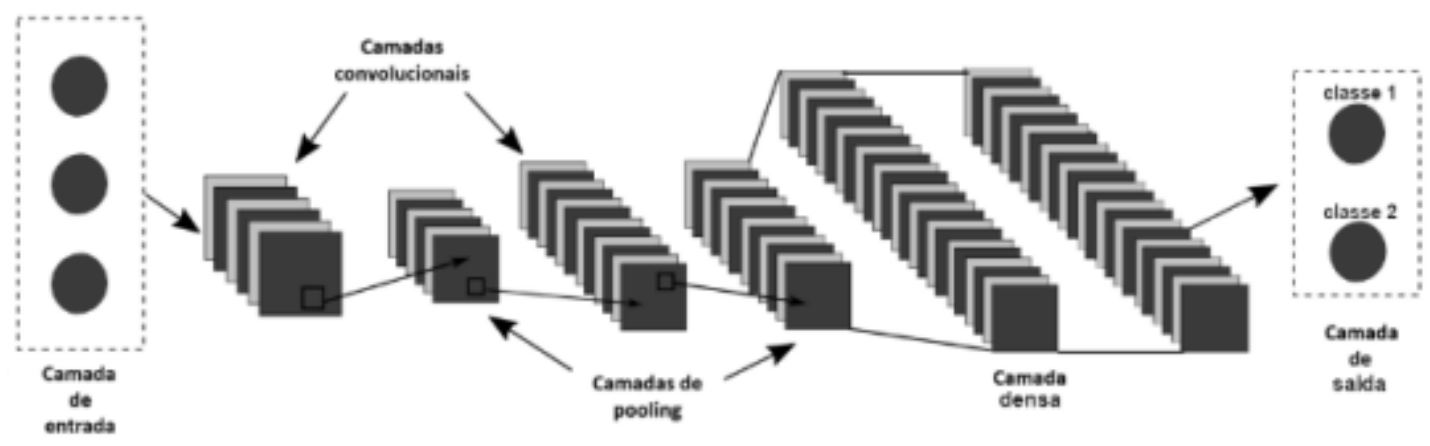

Figura 2. Arquitetura CNN. Fonte: [de Freitas et al. 2019].

A Figura 2 mostra as 5 principais camadas de uma Rede Convolucional, onde a camada de entrada é responsável por receber os padrões que serão apresentados para a CNN processar, na camada convolucional, cada neurônio utilizado como um filtro aplicado a uma imagem de entrada, onde o filtro é constituído por uma matriz de pesos. O 
pooling é uma forma de reduzir a dimensionalidade espacial dos mapas de características gerados pela rede. A camada densa é uma camada oculta, que por sua vez, cada neurônio possui um peso associado a cada elemento do vetor de entrada e por último, a camada de saída é responsável por receber os estímulos das camadas intermediárias e construir o padrão que vai constituir a resposta final.

\subsection{Arquitetura UNet}

A UNet é uma evolução da CNN tradicional, que foi projetada pela primeira vez em 2015 para processar imagens biomédicas. A arquitetura da UNet é ilustrada na Figura 3. O UNet consiste em três partes: downsampling, upsampling e skip connection e originalmente foi proposto por Ronneberger [Ronneberger et al. 2015]. A esquerda está o processo de compressão, ou seja, o codificador, no qual o modelo obtém uma imagem como entrada e aplica várias camadas de convolution, max-pooling e ReLU activation e compacta os dados em um espaço latente. No decodificador, a rede tenta decodificar as informações por meio de uma operação de convolução transposta (deconvolução) e produz a máscara de segmentação da imagem. No meio, os recursos obtidos no codificador são combinados com os recursos obtidos no decodificador por meio das skip connection e os recursos são refinados [Cai et al. 2021].

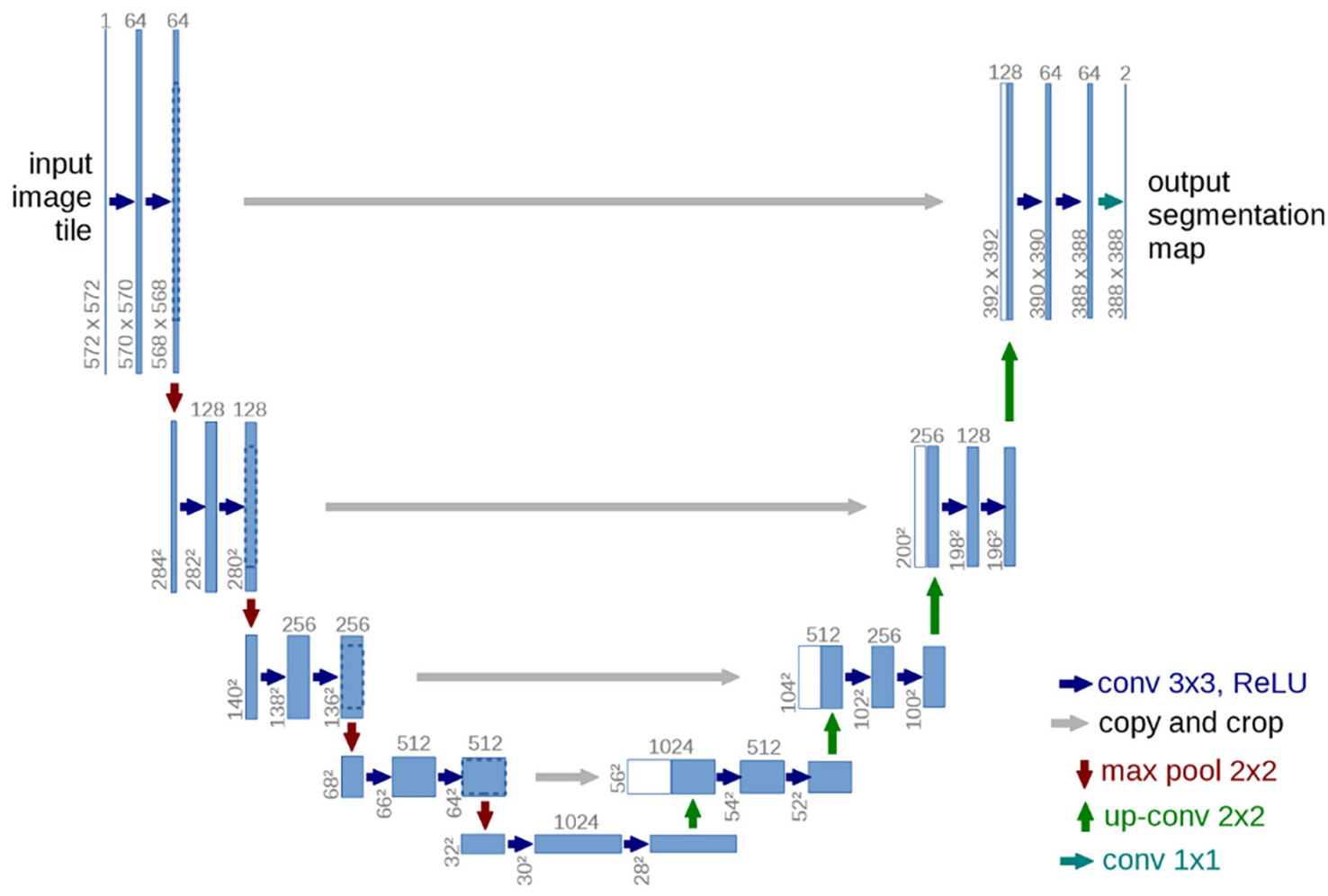

Figura 3. Arquitetura UNet.

Neste trabalho foi proposto uma arquitetura adaptada com cinco camadas de profundidade. Esta arquitetura de aprendizado profundo é simétrica e expansível por contração, permitindo capturar o contexto da imagem em sintonia com sua localização. Nas camadas da via de expansão, é inserido o operador up-sampling que vai redimensionar a imagem (ou copiar o pixel quantas vezes for necessário), portanto, é uma técnica de processamento digital que vai aumentar artificialmente a taxa de amostragem $N$ vezes, 
inserindo um número $N-1$ de zeros entre as amostras originais do sinal, e passando o conjunto obtido por um filtro de reconstrução, é importante frizar o fato que quanto maior a interpolação, mais a informação referente ao sinal original é distorcida ou perdida. Em seguida, vai ocorrer a concatenação entre camadas de contração e expansão.

As camadas relacionadas à contração consitem em camadas duplas de convolução, cadas uma delas acompanhadas de um Dropout que é utilizado para evitar que determinadas partes da rede neural tenham muita responsabilidade e consequentemente, possam ficar muito sensíveis a pequenas alterações, garantindo que o modelo seja robusto para a perda de qualquer evidência individual. Ele vem seguido do max-pooling onde o kernel extrai o valor máximo da área que ele convolve, coletando apenas a informação necessária. Quando é encontrado um recurso, sua exata localização não é tão importante quanto sua localização aproximada em relação a outros recursos. Um dos benefícios é que existem menos recursos agrupados e, por isso ajuda a reduzir o número de parâmetros necessários nas camadas posteriores. E por ultimo é utilizado a função de ativação Relu abreviação para rectified linear unit que por sua vez retorna 0 para todos os valores negativos, e o próprio valor para valores positivos. Como seu resultado é zero para valores negativos, ela tende a "apagar"alguns neurônios durante um passo forward, o que vai causar o aumento da velocidade do treinamento.

\section{Metodologia}

Inicialmente a tarefa mais importante é a compreensão do corpo lúteo obtido por meio do doppler. Tal tarefa consistiu em um estudo realizado em artigos e sites especializados, sendo que as características de tais imagens foram abordadas nas seções anteriores. Em seguida, é realizada a obtenção das imagens, sendo elas adquiridas em campo pelo NAPER. A etapa seguinte consiste na melhoria e normalização das imagens da base com o objetivo de remover informação desnecessária e melhorar o contraste. A etapa final é análise e segmentação das imagens com o objetivo de auxiliar o especialista na determinação automática da prenhez em vacas considerando a área de perfusão do corpo lúteo. Estas etapas serão descritas a seguir.

\subsection{Aquisição das imagens}

A aquisição foi realizada pelo NAPER durante atividades de campo. Foram geradas 159 imagens, a partir das quais também são fornecidas a versão com o doppler e uma versão delimitada realizada por especialista. Assim, as imagens são divididas em três subdiretórios: Delimitada, Doppler e Normal, totalizando 477 imagens. Elas possuem dimensões de 800x600 pixels, e formato.TIF. A Figura 4 mostra a equivalência das imagens nos três subdiretórios, sendo que à Figura 3(a) apresenta as imagens do diretório normal, à Figura 3(b) apresenta à imagem delimitada por especialista do NAPER e à Figura 3(c) mostra a imagem adquirida por meio de equipamento Doppler capaz de captar o fluxo sanguíneo, fazendo seu destaque em azul e vermelho. 


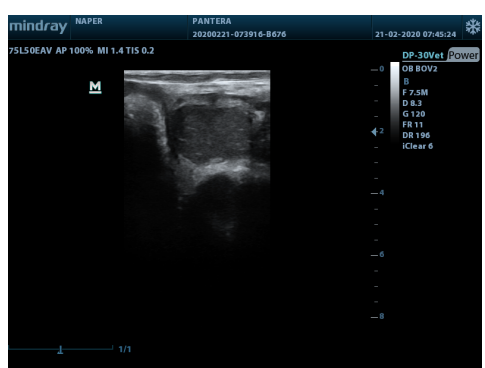

(a) Normal

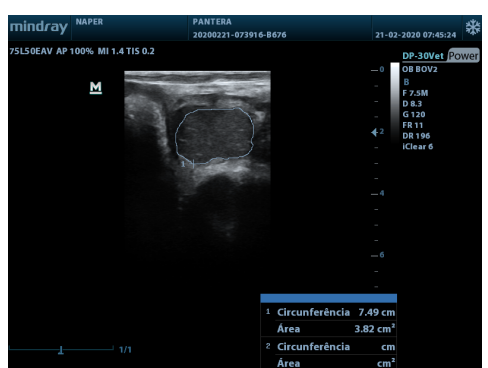

(b) Delimitada

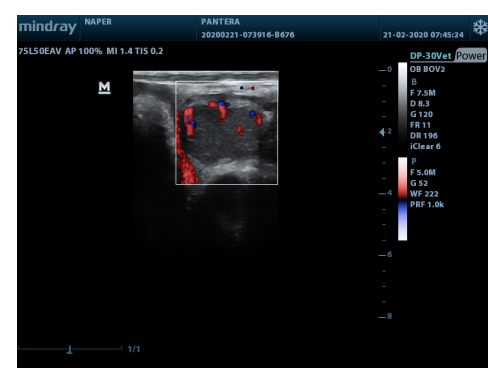

(c) Doppler

Figura 4. Equivalência das Imagens.

\subsection{Normalização e melhoria das imagens}

Foi observada a necessidade de melhorar as imagens em função do baixo contraste. Foram testadas duas funções de ajuste, a primeira testada foi o ajuste gamma: é gerada uma curva e a imagem tem seu contraste reorganizado conforme a curva, com isso, ocorre o ajuste dos valores de intensidade da imagem por meio de um mapeamento. Porém, os testes mostraram que o método não se adapta tão bem ao tipo de imagem em questão e por isso foi descartado.

O segundo método para melhoria do contraste foi baseado em equalização do histograma, o Contrast Limited Adaptive Histogram Equalization (CLAHE). Na equalização adaptativa do histograma, a imagem é separada em sub imagens e o valor de intensidade dos pixels é redistribuído melhorando o contraste local. No entanto, esse processo pode aumentar o ruído quando usado em imagens ruidosas, como imagens obtidas por doppler, assim, o CLAHE é uma extensão da equalização adaptativa do histograma e se aplica melhor ao propósito a ser alcançado: o algoritmo distribui os níveis de intensidade e limita a amplificação do ruído. Após a configuração dos parâmetros da função e realização dos testes, foi observado que ele melhorou a visualização das imagens, sendo possível analisar de forma mais clara a área de perfusão. Na Figura 5 é mostrada a comparação da imagem original o resultado da equalização de histograma.

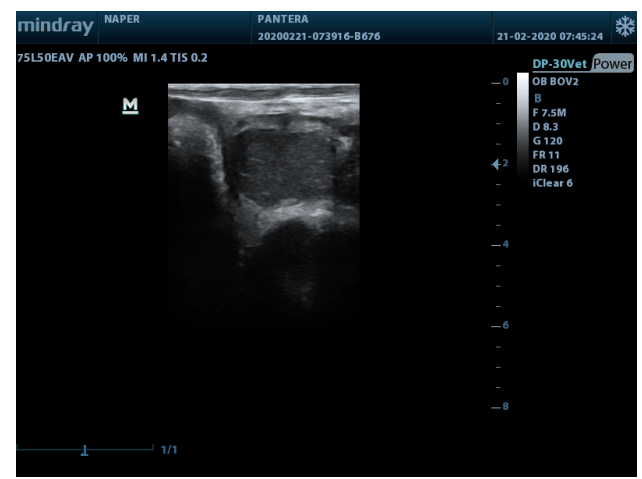

(a)

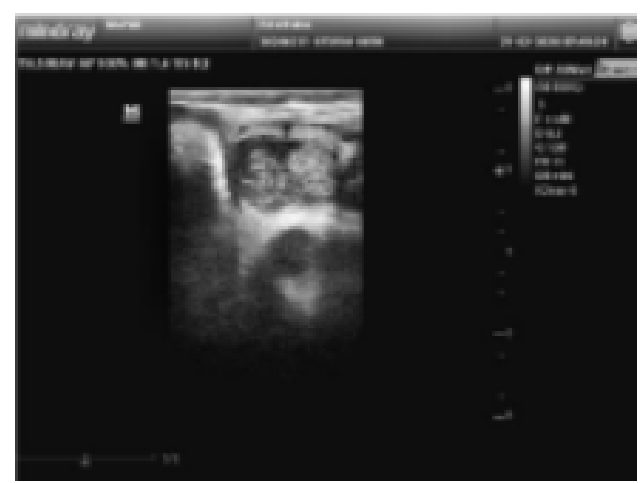

(b)

Figura 5. Equalização utilizando CLAHE. (a) Imagem Original. (b) Imagem Equalizada.

Na Figura 6 pode se observar a normalização realizada nas imagens, foi feito o 
recorte com a finalidade de reduzir a quantidade de dados a serem processados. Esse recorte foi feito de maneira automática em todas as imagens da base.

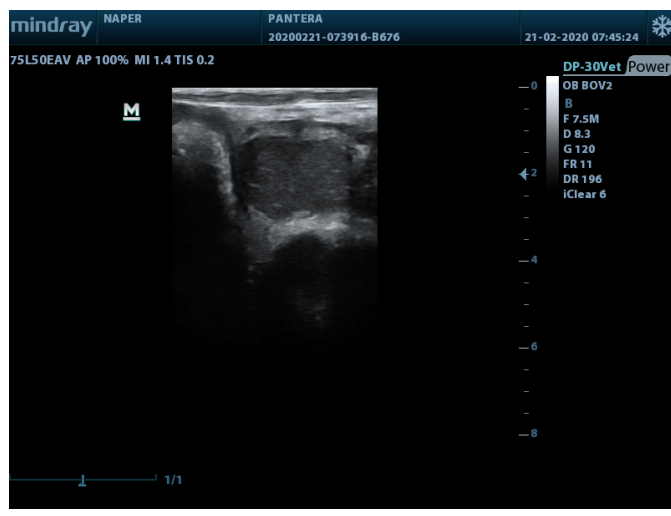

(a)

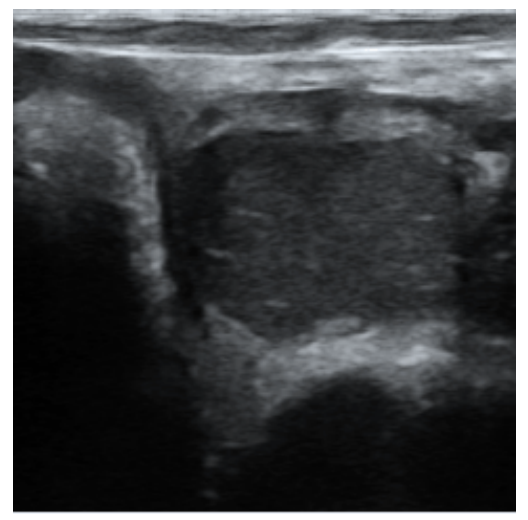

(b)

Figura 6. Normalização. (a) Imagem Original. (b) Imagem recortada.

\subsection{Segmentação das imagens}

Foi construída uma Convolutional Neural Network (CNN) com arquitetura Unet para a segmentação das imagens. Foi adotada a segmentação semântica, por meio de aprendizado supervisionado, em que a delimitação manual (Ground truth) realizada por um especialista foi fornecida para que a rede realize o aprendizado. As imagens foram separadas em dois conjuntos: testes e treinamento, $50 \%$ das imagens foram utilizadas para o treinamento e o restante para teste.

O ground truth precisou ser preparado para servir como entrada na rede. A Figura 7(a) mostra a segmentação realizada pelo especialista, enquanto que a Figura 7(b) mostra à binarização da imagem e a Figura 7(c) mostra o preenchimento da região de interesse na imagem.

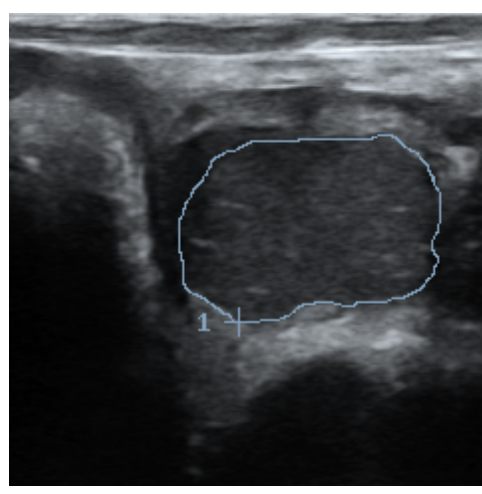

(a)

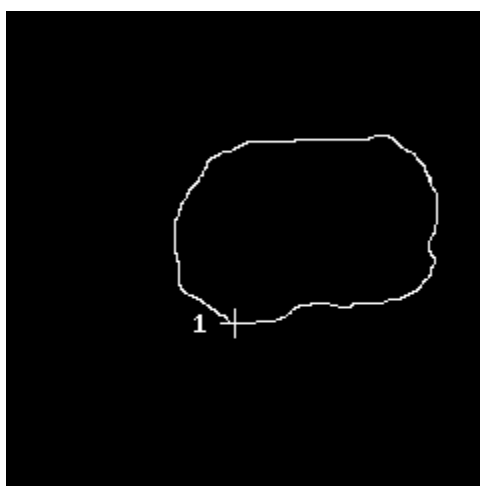

(b)

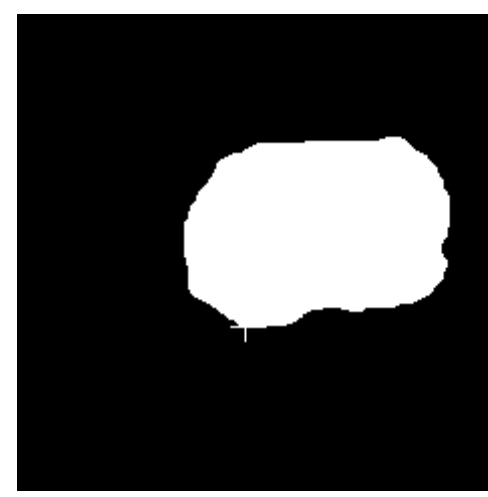

(c)

Figura 7. (a) Área de interesse determinada pelo especialista. / (b) Imagem Binarizada. / (c) Preenchimento da região de interesse. 


\section{Resultados}

A rede foi treinada com um número total de 140 imagens, sendo 70 para treinamento e 70 para testes, com uma resolução de $240 \times 240$ pixels e um número total de 300 épocas. O caráter de escolha das imagens utilizadas foi aleatório para se encaixar melhor no funcionamento da CNN. Foram avaliados cerca de 10 métricas, sendo elas: score, loss, accuracy, auc, precision, recall, true_positives, true_negatives, false_positivies e false_negatives. Após o treinamento da rede o melhor resultado com dados conhecidos (imagens de treinamento) e resultados com dados desconhecidos (imagens de testes) são mostrados na Tabela 1.

Tabela 1. Resultados dos dados de treinamento e teste.

\begin{tabular}{|l|l|l|l|l|}
\hline Dataset & Accuracy & AUC & Precision & Recall \\
\hline Treinamento & 0.9678 & 0.9763 & 0.9199 & 0.9199 \\
\hline Teste & 0.8847 & 0.9762 & 0.9198 & 0.9198 \\
\hline
\end{tabular}

Durante o treinamento da rede foi obtido 0.9678 de acurácia. Já nos testes foi obtido 0.8847 de acurácia. Portanto, é possível afirmar que a rede neural foi capaz de aprender a imagem do corpo lúteo das vacas, tornando possível o cálculo do nível de perfusão de maneira automática.

Também foi feita a contagem de pixels da área de perfusão, uma vez que este número é utilizado pelos especialistas do NAPER para verificar se a vaca está prenha ou não. A Figura 8 mostra um exemplo de contagem.
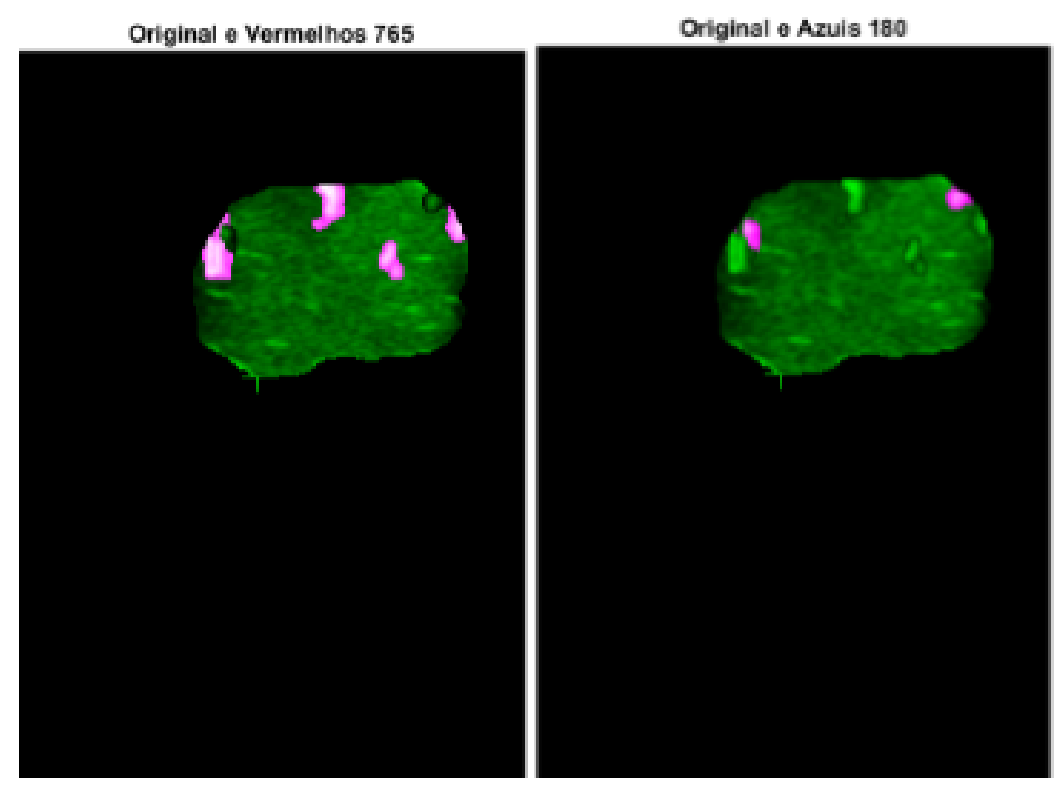

Figura 8. Contagem de Pixels Azuis e Vermelhos.

A Figura 9 apresenta a imagem de uma ultrassonografia de uma vaca gestante aos 21 dias de gestação. 


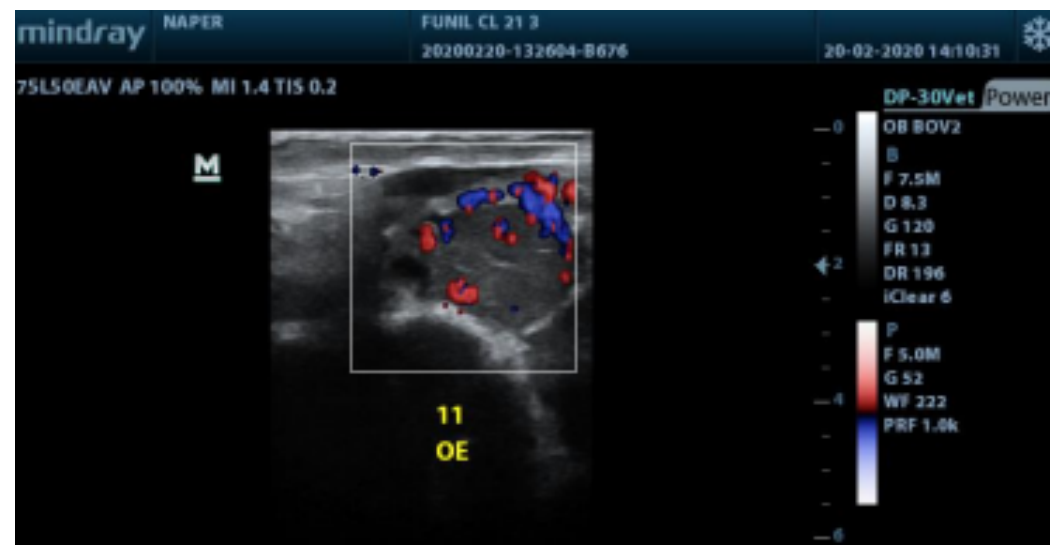

Figura 9. Perfusão de corpo lúteo de vaca gestante aos 21 dias.

Na figura 10 é apresentada a imagem com a perfusão do corpo lúteo de uma vaca não gestante aos 21 dias de gestação.

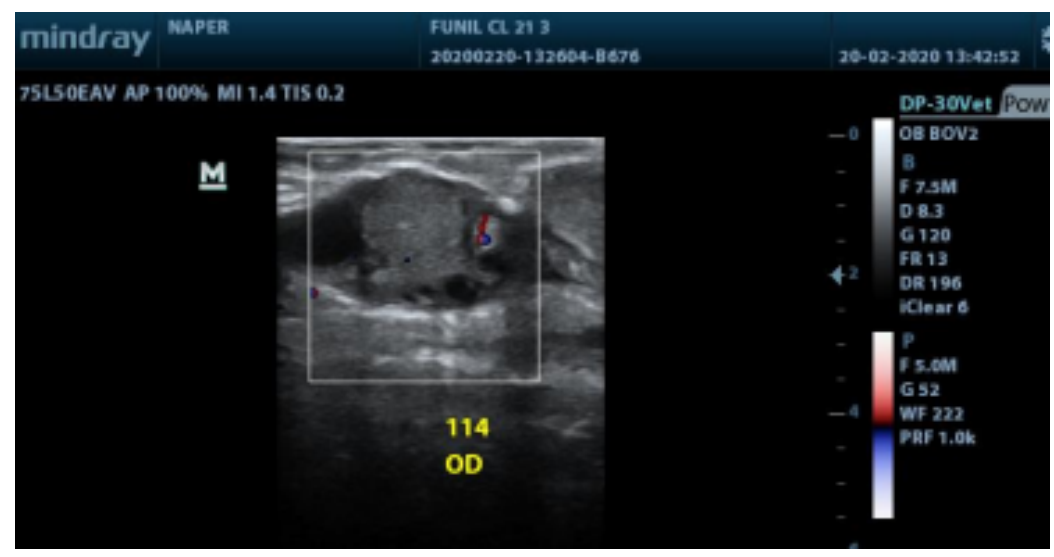

Figura 10. Perfusão de corpo lúteo de vaca não gestante aos 21 dias.

\section{Conclusão}

Diante dos resultados encontrados, é possível concluir que uma rede neural convolucional unida a técnicas de equalização do histograma se mostra muito eficiente na segmentação de imagens de ultrassom doppler do ovário de bovinos com a finalidade de obter o corpo lúteo. Consequentemente, é possível obter um método de determinação precoce e automático da gestação em bovinos, a apartir da avaliação da área de perfusão dentro da área delimitada pela rede. Neste caso, a gestação poderia ser detectada com 21 dias, uma vez que o método obteve uma acurácia de 0.9678 em dados conhecidos e 0.8847 em dados desconhecidos. Nesse sentido, a técnica pode ser utilizada em propriedades que visam maior eficiência produtiva, podendo assim diminuir intervalo entre partos e restabelecer uma ressincronização de forma antecipada a convencional. Como estudos futuros, será realizada a reconstrução das imagens e comparação da área de perfusão determinada pelo especialista e do método automático baseado em $\mathrm{CNN}$ e testes com outros métodos de melhoria de imagem com o intuito de aumentar o desempenho da rede. 


\section{Referências}

Cai, Y., Li, Q., Fan, Y., Zhang, L., Huang, H., and Ding, X. (2021). An automatic trough line identification method based on improved unet. Atmospheric Research, 264:105839.

Cheung, W., Stevenson, G. N., de Melo Tavares Ferreira, A. E. G., Alphonse, J., and Welsh, A. W. (2020). Feasibility of image registration and fusion for evaluation of structure and perfusion of the entire second trimester placenta by three-dimensional power doppler ultrasound. Placenta, 94:13-19.

de Freitas, D. S., da Silva Camargo, S., Comin, H. B., Domingues, R., Gaspar, E. B., and Cardoso, F. F. (2019). Reconhecimento da ceratoconjuntivite infecciosa bovina utilizando imagens termográficas e redes neurais convolucionais. Revista Brasileira de Computação Aplicada, 11(3):133-145.

Embrapa (2019). Diagnóstico precoce de prenhez com ultrassom Doppler em vacas ajuda a aumentar produção.

Gonzalez, R. C. and Woods, R. E. (2000). Processamento de imagens digitais. Editora Blucher.

Gonzalez, R. C. and Woods, R. E. (2010). Processamento digital de imagem. Pearson, ISBN-10: 8576054019, 10:11-27.

Kastelic, J., Curran, S., and Ginther, O. (1989). Accuracy of ultrasonography for pregnancy diagnosis on days 10 to 22 in heifers. Theriogenology, 31(4):813-820.

Ma, J., Fan, X., Yang, S. X., Zhang, X., and Zhu, X. (2017). Contrast Limited Adaptive Histogram Equalization Based Fusion for Underwater Image Enhancement. Preprints, (March):1-27.

Medeiros, M., Farinasso, A., Nery, I., Souza, H., Vasconcelos, J., Trindade, K., Batista, A., Manso, H., Carneiro, G., and Wischral, A. (2017). Estudo da vascularização folicular e do corpo lúteo de éguas cíclicas tratadas com extrato de pituitária equina utilizando ultrassom doppler colorido. Arquivo Brasileiro de Medicina Veterinária e Zootecnia, 69:1089-1096.

Pozor, M. and McDonnell, S. (2004). Color doppler ultrasound evaluation of testicular blood flow in stallions. Theriogenology, 61(5):799-810.

Pugliesi, G., Rezende, R. G., Silva, J. d., Lopes, E., Nishimura, T. K., Baruselli, P. S., Madureira, E. H., and Binelli, M. (2017). Uso da ultrassonografia doppler em programas de iatf e tetf em bovinos. Rev Bras Reprod Anim, 41(1):140-150.

Rodríguez Alvarez, J., Arroqui, M., Mangudo, P., Toloza, J., Jatip, D., Rodríguez, J. M., Teyseyre, A., Sanz, C., Zunino, A., Machado, C., and Mateos, C. (2018). Body condition estimation on cows from depth images using convolutional neural networks. Computers and Electronics in Agriculture, 155:12-22.

Rollim, V. M. (2017). Uso da ultrassonografia na reprodução de bovinos.

Ronneberger, O., Fischer, P., and Brox, T. (2015). U-net: Convolutional networks for biomedical image segmentation. In International Conference on Medical image computing and computer-assisted intervention, pages 234-241. Springer. 
Santos, A. M. d., Seixas, J. M. d., Pereira, B. d. B., and Medronho, R. d. A. (2005). Usando redes neurais artificiais e regressão logística na predição da hepatite a. Revista Brasileira de Epidemiologia, 8:117-126.

Trevisol, E, F. J. D. F. A. J. M. C. F. J. (2011). EFFECT ON CORPUS LUTEUM VOLUME AND VASCULARITY IN COWS AFTER OVULATION INDUCTION WITH DIFFERENT GNRH ANALOGUES. 